\section{A spectral luminosity function in the pigeon determined by flicker photometry ${ }^{1}$}

\author{
VIRGIL A. GRAF, Dartmouth College, \\ Hanover, N.H. 03755
}

A method was developed for using flicker photometry in determining photopic spectral sensitivity in the pigeon. The overall spectral curves for two animals were found to be in substantial agreement with previously published data, but the errors of measurement were reduced fourfold.

Flicker photometry has been used extensively with human Ss to determine photopic-luminosity functions. The advantages of the method are that it is highly reliable, the judgment required of $S$ is easy, and it is not necessary to use adaptation lights. For these reasons, Graf (1967) attempted to apply the method of flicker photometry to the study of spectral sensitivity in turtles. He found the method to be applicable to this lower animal over a limited spectral range. The results were reliable (standard error of measurement was 0.03 of a $\log$ unit), and the data agreed with electrophysiological measures of visual function in turtles obtained by several other Es (Armington, 1954; Granda \& Stirling, 1965). However, the appearance of several anomalies in the turtle data indicated that the method of flicker photometry should be tested with another species.

The purpose of this investigation was to use the method of flicker photometry with pigeons. Pigeons were chosen because there are extensive data on visual function from electrophysiology (Ikeda, 1965) as well as behavioral data on pigeon spectral sensitivity (Blough, 1957). The results from flicker photometry, therefore, can be compared to the results from these other lines of evidence.

\section{SUBJECTS}

The Ss were two White Carneaux pigeons obtained from the Palmetto Pigeon Farm, Sumpter, South Carolina. They were maintained at $85 \%$ of their ad lib feeding weight.

\section{APPARATUS}

The experimental chamber was a $28 \times 28 \times 31.5 \mathrm{~cm}$ enclosure placed inside a sound-attenuating picnic chest. The front panel contained a single translucent Plexiglas response key, $2.5 \mathrm{~cm}$ in diam, located $21.6 \mathrm{~cm}$ above the floor of the enclosure. The birds gained access to a conventional pigeon feeder through an aperture, $5.1 \times 6.4 \mathrm{~cm}$, located $8.9 \mathrm{~cm}$ above the floor.
The flicker photometer was similar to one previously described in detail (Graf, 1967). Briefly, a single 500-W projection lamp provided two independent beams. Each beam passed through polaroid material mounted orthogonally with respect to each other. The beams were combined with a beam splitter and passed through another piece of Polaroid material rotated by a feedback-controlled dc motor (Servo Dyne 4420, Cole-Palmer Corp., Chicago, IIl.). With this arrangement, each beam was modulated sinusoidally but $180 \mathrm{deg}$ out of phase. An image of a field lens in each beam was projected onto the response key with appropriate optics. The response key was painted flat black except for a circular patch, $1.3 \mathrm{~cm}$ in diam.

One beam of the flicker photometer was defined as the standard and had an average luminance of $.06 \mathrm{~mL}$. The other beam was defined as the variable and could be changed in luminance and spectral composition. Chromatic stimuli for the variable beam were obtained by passing the filament image of the source through a Schott interference wedge (Veril S-200), with a bandwidth of $15 \mathrm{~nm}$ for these conditions. Luminance of the variable beam was varied with Kodak neutral-density filters.

The stimulus that the pigeons observed consisted of the temporal alternation of each beam of the flicker photometer. Two alternation rates were used, $100 \mathrm{~Hz}$ and $10 \mathrm{~Hz}$.

The variable beam was calibrated in terms of the relative energy available at the wavelengths used. A silicone photocell was mounted inside the experimental chamber as close to the stimulus-response key as possible. The output of the photocell was led to a Keithly microammeter, and the current generated by the photocell was determined for each wavelength. The photocell sensitivity was calibrated with an Appley thermopile.

\section{PROCEDURE}

After conventional magazine and key-peck training, each animal was trained to discriminate high $(100 \mathrm{~Hz})$ and low $(13 \mathrm{~Hz})$ alternation rates when the standard beam was used alone. The assumption here was that high alternation rates appear "steady," and low alternation rates appear to "flicker."

A discrete-trials procedure was used for discrimination training. The animals were reinforced on a CRF schedule for responding to the "steady" light and extinguished for responding to the "flickering" light. If the pigeon responded to the steady light within $10 \mathrm{sec}$ after the onset of the trial, then the stimulus light was occluded and $S$ received access to grain for $3 \mathrm{sec}$. After a 10-sec intertrial interval (ITI), the next trial started. If S did not respond to the "steady" light within $10 \mathrm{sec}$, then the stimulus light was occluded and a 10-sec time-out period (TO) followed. Following the TO, the 10-sec ITI started, after which the trial was repeated. On "flickering" stimulus trials, the TO-ITI sequence was started, and the trial was repeated if the animal responded within $10 \mathrm{sec}$ of stimulus onset. If the pigeon did not respond for $10 \mathrm{sec}$, this was considered a correct response, the stimulus light was occluded, and the ITI was started. Thereafter, a new trial began. In no case was reinforcement given for response or nonresponse on "flicker" trials.

When the pigeons had learned to make the discriminative responses reliably $(90 \%$ or better for 2 consecutive days), a partial-reinforcement schedule was started. With the CRF schedule for response to the steady light, the animals received 60 trials per day and 30 reinforcements. On the final partial-reinforcement schedule, each animal received 180 trials per day and 30 reinforcements, with five reinforcements in each block of 30 trials The pigeons could not maintain the discrimination criterion on partial-reward schedules more stringent than this.

The next stage of training consisted of finding a luminance value for the chromatic variable that allowed the Ss to make reliable "steady-flicker" discriminations when the chromatic variable alternated with the standard beam. Once this luminance was determined, a test sequence was started the next day. The standard beam was kept at the luminance used during training, but the luminance of the chromatic beam was set at one of five different levels. Thus, on a typical day's testing session, there were 180 trials, 90 at high alternation rates and 90 at low alternation rates. On $100-\mathrm{Hz}$ trials, the animal was reinforced for responding to the lighted key equally often at each luminance level tested. On $13-\mathrm{Hz}$ trials, the animal was never reinforced.

Spectral sensitivity was determined using a criterion method. Psychometric functions of the probability of response on low-speed trials was plotted for each of the five luminance levels used. The function was U-shaped, that is, at low and high luminances of the variable, the animal could make the appropriate response (nonresponse) with a high degree of accuracy. At luminance values between these, discrimination was poor. The criterion was the midpoint of the psychometric function at the $80 \%$ correct 


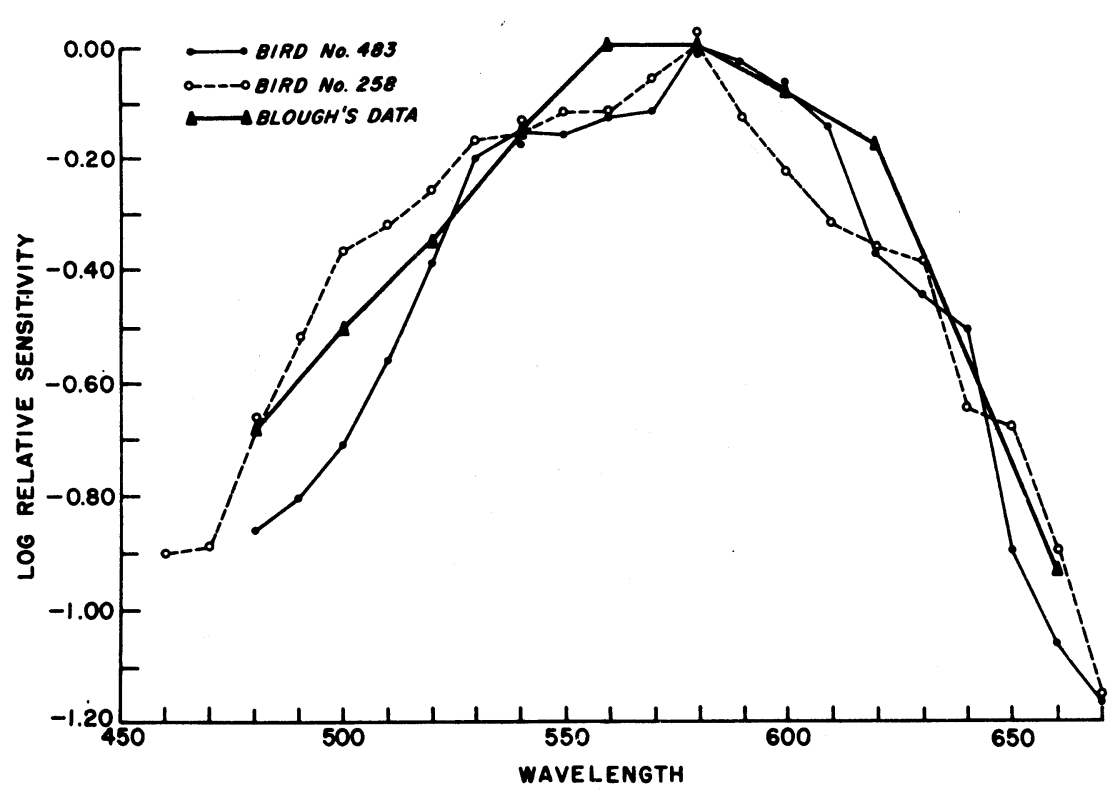

response level. Spectral sensitivity was taken as the logarithm of the reciprocal of the relative energy required to yield the criterion at each wavelength tested. Measurements were made at $10-\mathrm{nm}$ intervals from $460-670 \mathrm{~nm}$ for Bird No. 258 , and from $480-670 \mathrm{~nm}$ for Bird No. 483.

\section{RESULTS AND DISCUSSION}

The spectral sensitivity curves for the two pigeons are shown in Fig. 1. For comparison, Blough's data (1957) also are shown. It can be seen that there is substantial agreement in the sensitivity curves determined with Blough's tracking method and the flicker photometry results obtained in this experiment. It is likely that the processes underlying the sensitivity curves are the same in both cases.

One other comparison with Blough's psychophysical results can be made in regard to the reliability of the average sensitivity curves. The median standard error for Blough's data was 0.148 of a $\log$ unit calculated from the average data for four pigeons, whereas with flicker photometry data, the median standard error was only 0.035 of a $\log$ unit. This represents approximately a fourfold advantage of flicker photometry over the tracking method.

The standard error of measurement for each pigeon at each wavelength also was determined. This provides an estimate of the reliability of the individual bird's sensitivity. The errors of measurement for Bird No. 258 ranged from 0.003 to 0.2 of a $\log$ unit with a median of 0.06 of a $\log$ unit, and for Bird No. 483, from 0.01 to .09 of a $\log$ unit with a median of .05 of a $\log$ unit.

\section{reversal index in rats ${ }^{1}$}

\section{FRED P. VALLE, University of British Columbia, Vancouver 8, Canada}

Nineteen rats learned a spatial discrimination and reversal in a WGTA. There was a significant negative correlation between errors during initial learning and the reversal index $(r=-.523, p<.05), a$ significant positive correlation between errors during reversal learning and the reversal index $(r=+.679, p<.01)$, and $a$ low positive correlation between errors
Fig. 1. Relative spectral sensitivity of two pigeons determined with flicker photometry. The average data from four pigeons obtained with a tracking method (Blough, 1957) also are shown.

to perform selective-adaptation experiments using behavioral methods. If the overall spectral curves were partitioned in a manner congruent or similar to the electrophysiological curves, Ikeda's proposal would be on much firmer ground.

The data reported here indicate that flicker photometry is applicable to lower animals, and that the spectral curves obtained with this method are highly reliable. One additional advantage of this method is that the behavioral requirements for the animal are relatively simple, and they are easy to maintain.

\section{REFERENCES}

ARMINGTON, J. C. Spectral sensitivity in the turtle, Pseudemys. Journal of Comparative \& Physiological Psychology, 1954, 47, 1-6.

One approach that is commonly attempted with overall sensitivity curves is to speculate about possible underlying receptor systems. Ikeda (1965) performed such an analysis of his ERG data in relationship to his selective-adaptation data and Blough's overall spectral-sensitivity data. In brief, he concluded that the latter can be fitted by the simple average of two spectral curves determined by the ERG on-response (b wave) and the ERG off-response (d wave). The former has a peak at $547 \mathrm{~nm}$, whereas the latter peaks at $605 \mathrm{~nm}$. Although the data reported here are not inconsistent with this conclusion, it should be pointed out that the above set of spectral mechanisms do not exhaust the possible combinations that would yield a good fit to the overall spectral curve. One way to test Ikeda's conclusions would be
BLOUGH, D. S. Spectral sensitivity in the pigeon. Journal of the Optical Society of America, 1957, 47, 827-833.

GRAF, V. A spectral sensitivity curve and wavelength discrimination for the turtle, Chrysemys picta picta. Vision Research, 1967, 7, 915-928.

GRANDA, A. M. \& STIRLING, C. E. Differential spectral sensitivity in the optic tectum and eye of the turtle. Journal of General Physiology, 1965, 48, 901-917.

IKEDA, H. The spectral sensitivity of the pigeon (Columba livia). Vision Research, 1965, 5, 19-36.

\section{NOTES}

1. This research was supported by Grant NB MH 07222 from the National Institutes of Health, USPHS.

2. The assumption about how pigeons actually "perceive" the lights is not necessary. It is simply easier to talk about "steady" and "flickering" lights than it is to talk about high and low alternation rates.

\section{Correlations between error scores and the}

during initial learning and errors during reversal learning $(r=+.143)$.

Warren (1967) has shown that, for naive cats, the size of the reversal index (ratio of errors during reversal learning to errors during initial learning; see Rajalakshmi \& Jeeves, 1965) is (1) significantly negatively correlated with numer of errors made during initial learning and (2) significantly positively correlated with number of errors 